\title{
CORRESPONDENCE OPEN \\ Recovered COVID-19 patients with recurrent viral RNA exhibit lower levels of anti-RBD antibodies
}

\author{
Bingfeng Liu', Yaling Shi ${ }^{2}$, Wanying Zhang ${ }^{1}$, Rong $\mathrm{Li}^{1}$, Zhangping $\mathrm{He}^{1}$, Xiaofan Yang ${ }^{1}$, Yuejun Pan ${ }^{2}$, Xilong Deng ${ }^{2}$, Mingkai Tan ${ }^{2}$, \\ Lingzhai Zhao ${ }^{2}$, Fan Zou ${ }^{1,3,4}$, Yiwen Zhang ${ }^{1}$, Ting Pan ${ }^{1}$, Junsong Zhang ${ }^{1}$, Xu Zhang ${ }^{1}$, Fei Xiao ${ }^{5}$, Fang $\mathrm{Li}^{2}$, Kai Deng $\mathbb{D}^{1}{ }^{1}$ and Hui Zhang ${ }^{1}$ \\ Cellular \& Molecular Immunology (2020) 17:1098-1100; https://doi.org/10.1038/s41423-020-00528-0
}

Coronavirus disease 2019 (COVID-19), caused by infection with severe acute respiratory syndrome coronavirus 2 (SARS-CoV-2), has rapidly become a global pandemic. Most discharge criteria for patients with COVID-19 recommend two consecutive negative reverse-transcriptase polymerase-chain reaction (RT-PCR) test results from respiratory specimens over a 24-hour interval. Reports are accumulating of recurrent viral RNA-positive (RP) nasopharyngeal or anal specimens from recovered patients in China, Italy, South Korea, and France. ${ }^{1-8}$ Two cohort studies reported that $14.5 \%(38 / 262)$ and $16.7 \%(69 / 414)$ of patients with COVID-19 retesting were positive for SARS-CoV-2 RNA after discharge. ${ }^{2,6}$ The potential causes of RP specimens may be related to several aspects, such as virological factors, including residual viral reservoir, intermittent viral release, tissue distribution or false negatives, as well as immunological and sampling methodological factors. ${ }^{3,9,10}$ Given that an increasing number of RP cases have been reported worldwide, this phenomenon cannot be simply ascribed to false-negative testing. It indeed occurs, as truly discharged patients could suffer reactivation or could be reinfected with SARS-CoV-2. ${ }^{3}$ The risk of possible retransmission still exists, and the serological characteristics remain largely unknown, which could jeopardize the management of recovered COVID-19 patients. ${ }^{11}$

In this study, we collected blood samples from 47 recovered patients. The median age of the patients was 49 years (range: 17-82 years), and $55.3 \%$ of them were women. According to the Chinese national treatment guidelines, their symptoms were classified as moderate (36 patients) or severe (11 patients), and the median disease duration (from the onset of illness to the last RNA-negative conversion before RP status) was 15 days (range: 1-40 days). Eight of the 47 recovered patients displayed RP anal or throat swab samples from 8 to 39 days after viral shedding, and 39 patients remained persistently RNA negative (PRN). None of the conditions of these RP patients were severe before recovery (Fig. S1a). The median disease duration was 16.5 days for RP patients (ranging from 10 to 24 days), which was not significantly different from that of PRN patients (15 days, ranging from 1 to 40 days) (Fig. S1b). Moreover, the RP patients were significantly younger than the PRN patients (median 34.5 years vs. 54 years, $p=0.019$ ), which is consistent with previous reports (Fig. S1c). ${ }^{1,2}$
Plasma samples were collected from these 47 patients with COVID-19 at the time of convalescence and assessed for antibodies against the following SARS-CoV-2 proteins: the spike glycoprotein (S); the receptor-binding domain (RBD); conserved heptad repeats (HR1-HR2) in the S2 domain; and the nucleocapsid (N), membrane $(\mathrm{M})$, and envelope $(\mathrm{E})$ proteins. The concentrations of IgG secreted in response to these SARS-CoV-2 proteins varied in different patients, with detection rates of $100.0 \%$ (47/47), 83.0\% (39/47), 97.9\% (46/47), $100.0 \%(47 / 47), 55.3 \%(26 / 47)$, and $21.3 \%$ (10/47) for the S, RBD, HR1-HR2, N, M, and E proteins, respectively (Fig. 1a; Fig. S2). The detection rates of IgM to the S, RBD, HR1-HR2, and N proteins were $100.0 \%$ (47/47), 95.7\% (45/47), 83.0\% (39/47), and 100\% (47/47), respectively (Fig. 1b; Fig. S2). Notably, significantly higher levels of SARS-CoV-2-specific IgG and IgM developed to the $S$ and $N$ proteins (Fig. 1a, b).

To evaluate the effect of specific antibodies on RP status, we compared the levels of anti-SARS-CoV-2 lgG to the $S, R B D$, $\mathrm{HR} 1-\mathrm{HR} 2, \mathrm{~N}$, and $M$ proteins in these patients during their convalescent period (Fig. 1c; Fig. S3). The results showed that RP patients induced significantly lower levels of anti-RBD IgG than PRN patients ( $p=0.013$ ) (Fig. 1c). As all of these RP patients were in a moderate condition before recovery, the PRN patients were further classified as moderate ( 28 patients) or severe ( 11 patients) according to their symptoms before recovery. The levels of antiRBD IgG in RP patients were still significantly lower than those of either PRN-severe or PRN-moderate patients $(p=0.012$ and $p=$ 0.040 , respectively; Fig. $1 d$ ). In addition, the patients with severe symptoms within the PRN group were more likely to induce higher levels of anti-RBD $\lg G(p=0.012$; Fig. 1d), which is consistent with previous reports. ${ }^{12}$ In contrast, there were no significant differences either in IgG to other viral proteins or in IgM between PRN and RP patients (Fig. 1c, e; Figs. S4, S5), suggesting that the humoral response to RBD rather than to other regions of the $S$ protein or the full-length $S$ protein might have played an important role in preventing viral rebound during recovery.

Furthermore, we observed that the titers of IgG to RBD among these recovered patients positively correlated with the spikebinding antibodies targeting the S, HR1-HR2, and N proteins $(r=$ $0.71, p<0.0001 ; r=0.53, p<0.0001 ;$ and $r=0.33, p=0.022$, respectively) but not with the $\mathrm{M}$ or E proteins (Fig. S6a). Moreover,

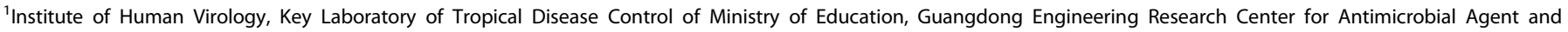

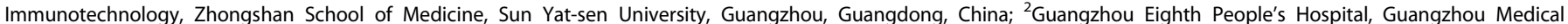

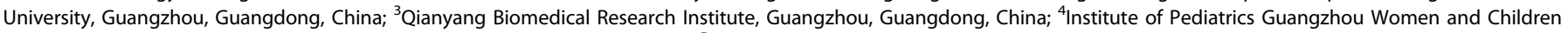
Hospital, Guangzhou Medical University, Guangzhou, Guangdong, China and ${ }^{5}$ Fifth Affiliated Hospital, Sun Yat-sen University, Zhuhai, Guangdong, China Correspondence: Fang Li (gz8hlf@126.com) or Kai Deng (dengkai6@mail.sysu.edu.cn) or Hui Zhang (zhangh92@mail.sysu.edu.cn)

These authors contributed equally: Bingfeng Liu, Yaling Shi, Wanying Zhang, Rong Li
}

Received: 28 July 2020 Accepted: 4 August 2020

Published online: 16 September 2020 
a

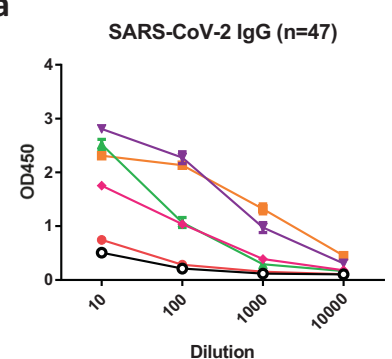

C

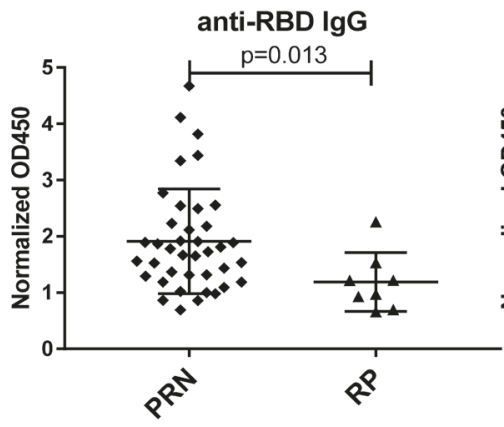

anti-N IgG

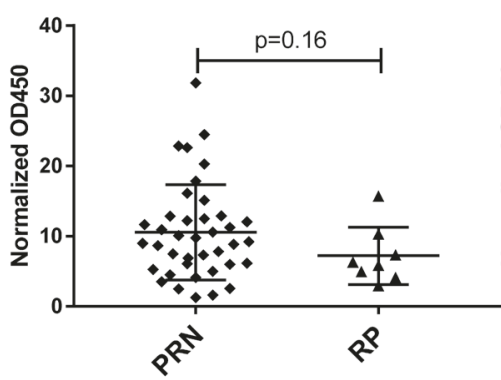

d

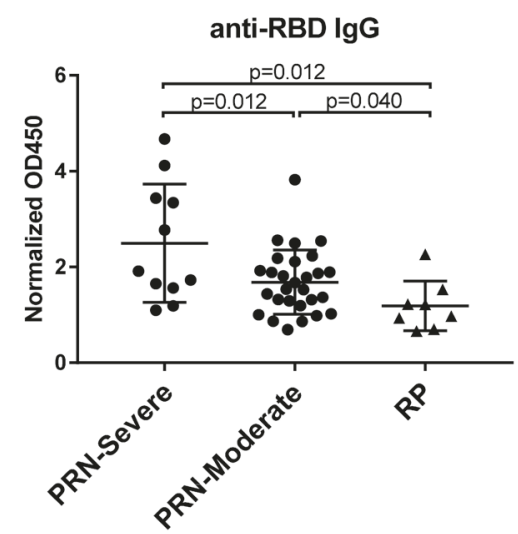

b
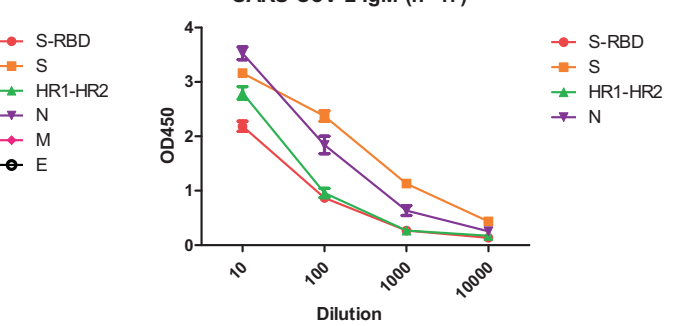

anti-S IgG

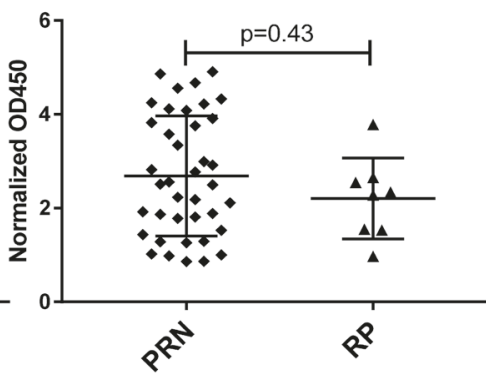

anti-M IgG
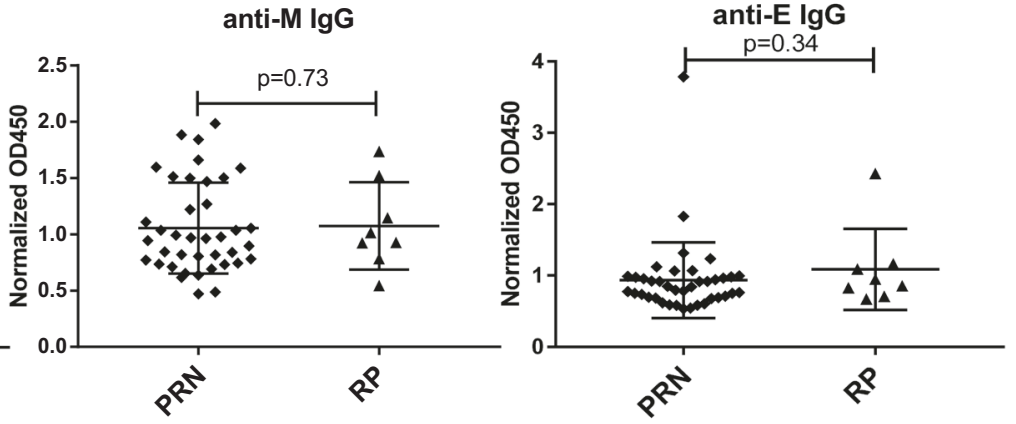

e

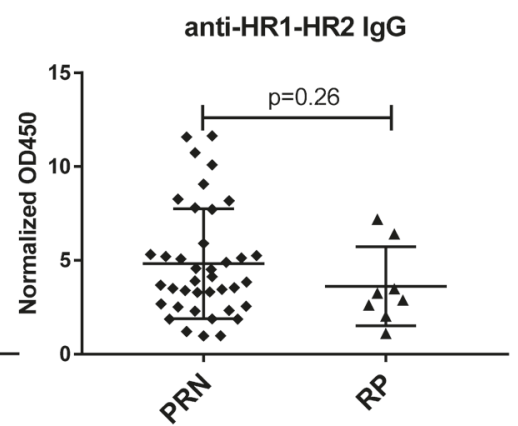

anti-E IgG

Fig. 1 Comparison of antibody responses to SARS-CoV-2 between PRN and RP patients. Kinetics of binding lgG (a) and lgM (b) targeting the $\mathrm{RBD}, \mathrm{S}, \mathrm{HR} 1-\mathrm{HR} 2, \mathrm{~N}, \mathrm{M}$, and E proteins in 47 recovered COVID-19-patient plasma samples collected during the convalescent period are shown. The $Y$-axis represents optical density units at OD450 $\mathrm{nm}$, and the $X$-axis represents reciprocal plasma dilutions. c Normalized OD450 nm values of the anti-SARS-CoV-2 lgG to the RBD, S, HR1-HR2, N, M and E proteins are compared between PRN and RP patients. The $P$ value was calculated using a two-tailed Mann-Whitney $U$ test or unpaired Student's $t$ test. d Normalized OD450 nm values of the anti-RBD IgG were compared between PRN-severe, PRN-moderate, and RP patients. The $P$ value was calculated using a two-tailed Mann-Whitney $U$ test or unpaired Student's $t$ test. e Normalized OD450 nm values of the anti-RBD IgM were compared between PRN and RP patients. The $P$ value was calculated using a two-tailed Mann-Whitney $U$ test 
the level of $\operatorname{lgM}$ to the RBD protein among these recovered patients also correlated with the S, HR1-HR2, and N proteins $(r=$ $0.67, p<0.0001 ; r=0.56, p<0.0001 ;$ and $r=0.60, p<0.0001$, respectively) (Fig. S6b). In addition, a positive correlation was also observed between age and IgG level to the RBD, S, HR1-HR2, and $\mathrm{N}$ proteins $(r=0.38, P=0.0077 ; r=0.40, P=0.0055 ; r=0.45, P=$ 0.0017 ; and $r=0.44, P=0.0021$, respectively; Fig. S7), indicating the important role of age in the generation of specific binding antibodies. $^{13}$

Because of the lack of clinical characteristics and the unknown significance of RP patients, it is critical to provide comprehensive serological profiling to guide the management of recovered COVID-19 patients after discharge. An important feature of the RP patients was their younger age than that of the PRN patients, and the ages of these recovered patients positively correlated with titers of IgG to the RBD protein. ${ }^{1,2,13}$ These observations are consistent with the conclusion that the level of IgG to the RBD protein in RP patients is significantly lower than that in the PRN group.

Based on our findings, the anti-RBD lgG level could serve as an indicator of RP status. To minimize the risk of possible viral rebound and retransmission during the current pandemic, close monitoring of anti-RBD lgG levels at viral shedding and a longterm follow-up of patients with lower levels of RBD antibodies is needed. Moreover, the relationship between anti-SARS-CoV-2 IgG titers and RP status suggests that the interplay between the virus and the host immune response in coronavirus infections should be further investigated for the development of more accurate diagnostic technologies and effective vaccines against viral infection.

\section{ACKNOWLEDGEMENTS}

This work was supported by the National Special Research Program of China for Important Infectious Diseases (2018ZX10302103 and 2017ZX10202102), the Special 2019-nCov Program of the Natural Science Foundation of China (NSFC) (82041002), the Important Key Program of the NSFC (81730060), and the Joint-Innovation Program in Healthcare for Special Scientific Research Projects of Guangzhou (201803040002) to H.Z. Further, the authors received support from the Natural Science Foundation of China (81672024), the Natural Science Foundation of Guangdong Province of China (2017A030306005), and the Guangdong Innovative and Entrepreneurial Research Team Program (2016ZT06S638) to K.D.; the Department of Science and Technology of Guangdong Province (2020B111108001) to F.L.; and the National Natural Science Foundation of China (81701989) to B.L.

\section{AUTHOR CONTRIBUTIONS}

B.L., Y.S., W.Z., and R.L. designed the experiments, performed most of these experiments, analyzed the data, and wrote the paper. Z.H. and X.Y. performed some of the experiments. Y.P., X.D., M.T., and L.Z. contributed to patient recruitment, data collection, and clinical management. F.Z., Y.Z., T.P., J.Z., X.Z., and F.X. provided scientific expertise and the interpretation of data for the work. F.L., K.D., and H.Z. had the idea for and designed the study, had full access to all data in the study and take responsibility for the integrity of the data and the accuracy of the data analysis. All authors reviewed and approved the final version of the report.

\section{ADDITIONAL INFORMATION}

The online version of this article (https://doi.org/10.1038/s41423-020-00528-0) contains supplementary material.

Competing interests: The authors declare no competing interests.

\section{REFERENCES}

1. Lan, L. et al. Positive RT-PCR test results in patients recovered from COVID-19. Jama 323, 1502-1503 (2020).

2. An, J. et al. Clinical characteristics of the recovered COVID-19 patients with redetectable positive RNA test. medRxiv https://doi.org/10.1101/2020.03.26.200 44222 (2020).

3. Hoang, V. T., Dao, T. L. \& Gautret, P. Recurrence of positive SARS-CoV-2 in patients recovered from COVID-19. J. Med .Virol. https://doi.org/10.1002/jmv.26056 (2020).

4. Chen, J. et al. Clinical course and risk factors for recurrence of positive SARS-CoV2 RNA: a retrospective cohort study from Wuhan, China. medRxiv https://doi.org/ 10.1101/2020.05.08.20095018 (2020).

5. Kipkorir, V., Cheruiyot, I., Ngure, B., Misiani, M. \& Munguti, J. Prolonged SARS-Cov2 RNA detection in anal/rectal swabs and stool specimens in COVID-19 patients after negative conversion in nasopharyngeal RT-PCR Test. J. Med. Virol. https://doi. org/10.1002/jmv.26007 (2020).

6. Huang, J. et al. Recurrence of SARS-CoV-2 PCR positivity in COVID-19 patients: a single center experience and potential implications. medRxiv https://doi.org/ 10.1101/2020.05.06.20089573 (2020).

7. Mei, Q. et al. Assessment of patients who tested positive for COVID-19 after recovery. Lancet Infect. Dis. https://doi.org/10.1016/s1473-3099(20)30433-3 (2020).

8. Loconsole, D. et al. Recurrence of COVID-19 after recovery: a case report from Italy. Infection, 1-3, https://doi.org/10.1007/s15010-020-01444-1 (2020).

9. Wölfel, R. et al. Virological assessment of hospitalized patients with COVID-2019. Nature https://doi.org/10.1038/s41586-020-2196-x (2020).

10. Ni, L. et al. Detection of SARS-CoV-2-specific humoral and cellular immunity in COVID-19 convalescent individuals. Immunity https://doi.org/10.1016/j. immuni.2020.04.023 (2020).

11. Wu, J. et al. Coronavirus disease 2019 test results after clinical recovery and hospital discharge among patients in China. JAMA Netw. Open 3, e209759, https://doi.org/10.1001/jamanetworkopen.2020.9759 (2020).

12. Tan, W. et al. Viral kinetics and antibody responses in patients with COVID-19. medRxiv https://doi.org/10.1101/2020.03.24.20042382 (2020).

13. Wu, F. et al. Neutralizing antibody responses to SARS-CoV-2 in a COVID-19 recovered patient cohort and their implications. medRxiv https://doi.org/10.1101/ 2020.03.30.20047365 (2020).

Open Access This article is licensed under a Creative Commons Attribution 4.0 International License, which permits use, sharing, adaptation, distribution and reproduction in any medium or format, as long as you give appropriate credit to the original author(s) and the source, provide a link to the Creative Commons license, and indicate if changes were made. The images or other third party material in this article are included in the article's Creative Commons license, unless indicated otherwise in a credit line to the material. If material is not included in the article's Creative Commons license and your intended use is not permitted by statutory regulation or exceeds the permitted use, you will need to obtain permission directly from the copyright holder. To view a copy of this license, visit http://creativecommons. org/licenses/by/4.0/.

(c) The Author(s) 2020 